\title{
Aptitud de padres para prevenir abuso sexual en menores después de una intervención educativa participativa
}

\author{
Martha Alicia Higareda-Almaraz, D en CM, (I) Enrique Higareda-Almaraz, M en Pedag, (2) \\ Irma Reyna Higareda-Almaraz, M en Sexol, (3) Juan Carlos Barrera-de León, (I) \\ Meynardo Alonso Gómez-Llamas, ${ }^{(2)}$ Verónica Benites-Godínez, D en Farmacol.(4)
}

\author{
Higareda-Almaraz MA, Higareda-Almaraz E, \\ Higareda-Almaraz M, Barrera-de León JC, \\ Gómez-Llamas MA, Benites-Godínez V. \\ Aptitud de padres para prevenir abuso sexual \\ en menores después de una intervención \\ educativa participativa. \\ Salud Publica Mex 20II;53:I34-I40.
}

\begin{abstract}
Resumen
Objetivo. Evaluar la aptitud en los padres sobre el impacto de educar con equidad a los menores, para prevenir abuso sexual infantil con una estrategia educativa participativa. Material y métodos. Diseño cuasiexperimental. Se incluyó a 92 padres con hijos menores en preescolar que recibieron una intervención educativa con estrategia promotora participativa por una hora durante 20 días. Para evaluar el cambio de aptitud se construyó previamente un cuestionario estructurado con 20 enunciados, que fue validado por expertos en educación y sexología infantil. Para comparar la diferencia intragrupos se utilizóWilcoxon. Resultados. Se encontraron diferencias estadísticamente significativas en las respuestas de los padres antes/después de la intervención educativa con un valor en la mediana (rango) I0(2-I2)/I8(6-20), $p<0.01$. Conclusión. Después de la intervención educativa participativa, se encontró un cambio de aptitud en los padres al adquirir un mayor aprendizaje sobre el impacto de educar con equidad a los menores para prevenir abuso. Es necesario continuar evaluando diferentes estrategias educativas.
\end{abstract}

Palabras clave: abuso sexual infantil; educación sexual; padres; equidad; México
Higareda-Almaraz MA, Higareda-Almaraz E, Higareda-Almaraz M, Barrera-de León JC, Gómez-Llamas MA, Benites-Godínez V.

Parental aptitude to prevent child sexual abuse after a participatory education intervention.

Salud Publica Mex 20II;53:134-140.

\begin{abstract}
Objective. To evaluate the aptitude of parents regarding the educational impact of equity education for children to prevent child sexual abuse using participatory strategies. Material and Methods. Quasi-experimental design. Ninetytwo parents with children in preschool were included in the study. The parents were given a course using participatory educational strategies for one hour daily over a period of 20 days. Prior to the course, a group of experts in child education and sexology prepared a questionnaire with 20 sentences. A Wilcoxon test was used to compare intergroup differences Results. We found statistically significant differences in the parents' responses before and after the educational intervention, with a median (range) of $10(2-12) / 18(6-20)$, $\mathrm{p}<0.01$. Conclusions. A significant change in aptitude was noted when parents attended classes using a participatory strategy to learn about the impact of educational equity for the prevention of child sexual abuse. Thus, it is imperative to continue evaluating different educational strategies.
\end{abstract}

Key words: child sexual abuse; sex education; parents; equity; Mexico

(I) Hospital Ginecología y Obstetricia, Centro Médico Nacional de Occidente, Instituto Mexicano del Seguro Social. Guadalajara, México.

(2) Jefe de Enseñanza del HGZ I, Instituto Mexicano del Seguro Social. Tepic, Nayarit, México.

(3) Hospital de Salubridad. Grullo, Jalisco, México.

(4) Coordinadora Delegacional de Enseñanza en Salud de Nayarit. Nayarit, México.

Fecha de recibido: 9 de agosto de 2010 - Fecha de aceptado: 10 de marzo de 201 I Solicitud de sobretiros: Dra. Martha Alicia Higareda Almaraz. Prisciliano Sánchez 530. 44100, Guadalajara, Jalisco. Correo electrónico: marthahigaredal2@hotmail.com. 
$\mathrm{E}^{\mathrm{L}}$ abuso sexual infantil (ASI) es frecuente en todo el mundo. Aproximadamente 150 millones de niñas y 73 millones de niños han sido víctimas de abuso sexual antes de los 18 años. ${ }^{1}$ Este abuso ocasiona daño físico y psicológico, generando conflictos en la familia y en la sociedad. ${ }^{2-8}$

El ASI es una forma de maltrato. Algunos autores lo definen como una interacción sexual que puede o no ser física entre un niño y un individuo, quien lo utiliza en forma violenta con engaño o seducción para su estimulación sexual, la del menor o la de otro. El niño no tiene la capacidad para dar consentimiento para este tipo de abuso. ${ }^{8,9}$ En el ASl se encuentran dos tipos de personalidades, la del agresor, el cual tiene mayor edad o madurez y es quien abusa de su poder contra la víctima. ${ }^{10}$

En la formación de la personalidad influyen varios factores ambientales que se encuentran en la familia, cultura y sociedad, que estimulan a los sujetos e interactúan con la genética individual, desarrollando diferentes personalidades. ${ }^{11}$ Dentro de la familia se han descrito varios factores que pueden ser un estímulo para formar la personalidad pasivo-agresiva, entre los que se encuentra el antecedente de maltrato físico, emocional, negligencia, abandono y testigos de violencia durante la infancia. ${ }^{12}$

Algunas características presentes en los menores pueden influir en algunos padres para maltratarlos, entre estas se encuentra la edad, y género, alguna discapacidad o enfermedad presente, ser un hijo no deseado, ser adoptado o tener hiperactividad. ${ }^{13}$ En algunas ocasiones, estas características influyen en los padres para impartir una educación con inequidad a los menores, otorgando el poder a uno de sus hijos; esto puede contribuir a formar la personalidad de un futuro agresor, en otras ocasiones al menor se le discrimina o daña, convirtiéndolo en una persona sumisa, desarrollando la personalidad de víctima. ${ }^{8-14,15}$

Algunos autores describen la importancia sobre la equidad de género para evitar formar mujeres que acepten la violencia, convirtiéndolas en víctimas. ${ }^{16}$ Otros autores describen la importancia de la igualdad de género y educación para mejorar la salud de la mujer en todo el mundo. ${ }^{17}$

Una de las medidas para cambiar la aptitud en las personas es la educación. Existen diferentes pruebas para evaluar la aptitud, entre las que se encuentra la del razonamiento verbal. Esta valora la habilidad para comprender temas específicos, deducir información y llegar a conclusiones relevantes por medio de la comprensión de los temas.

La mayoría de las intervenciones educativas sobre ASI son dirigidas a niños. La enseñanza se basa en desa- rrollar habilidades para identificar situaciones peligrosas con identificación del agresor y saber pedir ayuda. El material utilizado para el aprendizaje incluye videos, audiovisuales, pláticas y representaciones teatrales. ${ }^{18-21}$ En estas intervenciones educativas los menores tienen una participación pasiva y el aprendizaje se almacena en la memoria, por lo que puede ser olvidado con el tiempo.

Algunos autores describen que para lograr un mayor aprendizaje es importante la participación activa de los alumnos. En la intervención educativa con estrategia promotora de la participación el alumno elabora y transforma la información con base en la crítica y la autocrítica. Este proceso educativo pretende el perfeccionamiento en la calidad del aprendizaje para resolver problemas y tomar mejores decisiones. ${ }^{22-24}$

El propósito de este estudio fue evaluar la aptitud en los padres antes y después de una intervención educativa con la estrategia promotora participativa, sobre el tema impartido: impacto de educar con equidad a los menores para prevenir abuso sexual infantil

\section{Material y métodos}

El presente estudio fue conducido de enero a diciembre de 2009 en la escuela de educación preescolar Niños Héroes en Autlán, Jalisco, México. Se empleó un diseño del estudio cuasiexperimental.

De acuerdo con los criterios de inclusión y exclusión, fueron incluidos los padres o tutores que tenían hijos en educación preescolar. Fueron excluidos los que no presentaron la evaluación a través de un cuestionario estructurado, antes y después de la intervención educativa, y los que no tuvieron $100 \%$ de asistencia al curso-taller sobre la importancia de educar a los hijos con equidad para prevenir abuso sexual infantil.

Se consideró un cambio de aptitud cuando se encontró un mayor aprendizaje. Éste se evaluó con el incremento en el puntaje de las respuestas en relación con el valor basal con diferencias estadísticamente significativas.

Para el tamaño de la muestra fueron seleccionados todos los padres con menores en preescolar de la escuela donde se realizó la investigación, y se utilizó un muestreo consecutivo.

Para el desarrollo del estudio se solicitó la autorización de la inspección general de la Secretaría de Educación Pública de la localidad para evaluar una intervención educativa con estrategia promotora de la participación. Los participantes fueron los padres o tutores con menores en educación preescolar. Para evaluar el cambio de aptitud se elaboró un cuestionario estructurado. 


\section{Instrumento de medición}

a) Construcción. Se construyó un instrumento de medición sobre el tema impartido: la importancia de una educación con equidad para prevenir ASI. Se realizaron inicialmente 50 enunciados para definir el abuso sexual infantil, conocer su frecuencia, identificar algunos factores para el desarrollo de la personalidad del agresor y de la víctima, la influencia de la familia para el desarrollo de estas personalidades, las estrategias educativas utilizadas para prevenir este tipo de abuso y la importancia de educar con equidad a los menores para prevenir el abuso sexual infantil.

b) Validación. 1) Los enunciados se pasaron a ronda de cinco expertos en el campo de la educación y de la sexología educativa infantil para valoración de contenido y criterio; 2) se incluyeron las preguntas que tuvieron un consenso de los cinco expertos, dejando las más representativas y pertinentes, eliminando las confusas o inadecuadas; en total fueron 20 enunciados; 3) se realizaron ajustes y correcciones atendiendo al grado de dificultad para que correspondiera el nivel que se evaluó.

c) Confiabilidad. Se aplicó el cuestionario a un grupo piloto de 30 padres de familia, los cuales no participaron en el estudio. Para determinar la confiabilidad del instrumento se utilizó el coeficiente alfa de Cronbach, y se encontró un valor de 0.89 .

d) Método para calcular las calificaciones por el azar. El instrumento contó con tres opciones de respuestas: falso, verdadero y no sé, con las que se calificó una respuesta correcta +1 , la incorrecta y ausencia de respuesta -1 y la respuesta "no sé" $=0$. El máximo puntaje fue de 20. Se construyó una escala basada en el puntaje. Éste fue obtenido de las respuestas después de la intervención educativa en los padres: Muy alto (17-20), alto (14-16), media (11-13), bajo (8-10) y muy bajo (4-7).

\section{Procedimiento de evaluación}

Inicialmente se les informó a los padres sobre el tema que sería impartido: el impacto de una educación con equidad a los menores para prevenir el abuso sexual infantil. La asistencia fue voluntaria. Posteriormente se aplicó la intervención educativa (estrategia promotora de la participación).

1) Inicialmente se entregó a los padres copias de los temas sobre formación de la personalidad, desarrollo y educación de la sexualidad humana, y responsabilidad de los padres en la educación de los hijos. Estas copias fueron obtenidas del manual de orientación familiar editado por la Secretaría de Educación en Jalisco. Además se les solicitó a los padres que quisieran participar en buscar más información y compartirla en el grupo, siempre dirigidos por un facilitador.

2) Se formaron 16 equipos con cinco padres y dos equipos con seis padres. Cada equipo intercambió experiencias y conocimientos, y se estableció una discusión hasta lograr la comprensión. Finalmente se realizaron conclusiones.

3) Después de que cada equipo terminó el proceso anterior, se impartieron algunos temas sobre abuso sexual infantil: definición, frecuencia, factores de riesgo que influyen en la formación de la personalidad del agresor y de la víctima, medidas preventivas utilizadas y la importancia de educar con equidad a los menores para prevenir el abuso sexual infantil. Estos fueron impartidos por un facilitador especialista en sexología educativa infantil con una participación activa por parte de los padres de familia.

4) Posteriormente a la exposición se estableció una discusión por todo el grupo hasta lograr la comprensión de todos los participantes y se finalizó elaborando conclusiones.

\section{Descripción de los indicadores}

1. Abuso sexual infantil, tres enunciados: a) conocer la frecuencia y saber identificar las personalidades presentes: b) agresiva y c) de víctima.

2. Formación de la personalidad de los menores, dos enunciados: a) en los primeros años de la vida, b) los padres de los menores influyen en la formación de la personalidad.

3. Personalidades presentes en abuso sexual infantil y su repercusión, tres enunciados: a) agresor, b) víctima, c) daño físico y psicológico

4. Descripción de algunos conceptos sobre educación con equidad, cuatro enunciados: todos los hijos tienen derecho a lo siguiente: a) estudiar, b) realizar las mismas actividades, c) tener las mismas obligaciones, d) recibir las mismas sanciones.

5. Identificación de características de los menores que pueden influir en algunos padres para no educar con equidad, ocho enunciados: a) género, b) edad, c) presencia de alguna enfermedad crónica, d) tener alguna discapacidad manifestada por alguna alteración en el lenguaje, motricidad visual, auditiva, sensitiva u otra alteración que le impida realizar algunas actividades, e) adoptado, f) no deseado, g) belleza (color de la piel y ojos), h) cultura. 


\section{Mediciones}

Se llevó a cabo una medición antes y después de la intervención educativa en el grupo de estudio.

Análisis estadístico. Se realizaron frecuencias y porcentajes para variables cualitativas. Se utilizaron medianas y rangos para variables cuantitativas. Para comparar la diferencia entre porcentajes se utilizó ji². Para comparar la diferencia de medianas en las respuestas antes-después se empleó la prueba de Wilcoxon. Para realizar el análisis de los datos se utilizó el programa SPSS 13.0.

Ética. El protocolo fue aprobado por el Comité de Ética e Investigación del Hospital General de Zona número 1 en Tepic, Nayarit (número de aprobación R-2010-18025). El estudio fue desarrollado bajo las premisas de la declaración de Helsinki. ${ }^{25}$ Las personas seleccionadas para este estudio firmaron un consentimiento informado antes de ser incluidos dentro del estudio.

\section{Resultados}

Se analizó el cambio de aptitud en 92 padres con menores en preescolar a través de las respuestas antes y después de la intervención educativa con la estrategia promotora de la participación.

En el cuadro I se muestran las características de los padres que participaron en esta investigación. La mayoría fue del género femenino ( $98.9 \%$ ) con una media de 20 años de edad y estudios de secundaria (39\%)

\section{Cuadro I}

Características de los padres Que recibieron UNA INTERVENCION EDUCATIVA CON ESTRATEGIA PROMOTORA DE LA PARTICIPACIÓN PARA PREVENIR ABUSO SEXUAL INFANTIL. JaLISCO, MÉXICO, Diciembre de 2009

Característica

Padres con menores en preescolar $n=92$

\begin{tabular}{lc} 
Edad, mediana (rango) & $20(20-65)$ \\
$\begin{array}{l}\text { Género Mujeres, } \mathrm{n}(\%) \\
\text { Hombres, } \mathrm{n}(\%)\end{array}$ & $91(98.9)$ \\
& $\mathrm{I}(\mathrm{I} .1)$ \\
$\begin{array}{cc}\text { Escolaridad: Ninguna, } \mathrm{n}(\%) \\
\text { Primaria, } \mathrm{n}(\%)\end{array}$ & $\mathrm{I}(\mathrm{I} .10)$ \\
\hline Secundaria, $(\%)$ & $23(25.00)$ \\
\hline Bachillerato, $(\%)$ & $39(42.40)$ \\
\hline Licenciatura, $\mathrm{n}(\%)$ & $18(19.60)$ \\
\hline Posgrado, $\mathrm{n}(\%)$ & $10(10.90)$ \\
\hline
\end{tabular}

Nota: Se utilizaron frecuencias y porcentajes para variables; cualitativas, mediana y rango para la variable cuantitativa
En el cuadro II se encontró un mayor aprendizaje con cambio de aptitud en los padres después de la intervención educativa con la EPP sobre el tema impartido: El impacto de educar con equidad a los menores para prevenir abuso sexual infantil. Esto se demostró en las respuestas antes/después; se incrementó el valor de la mediana (rango) después de la intervención educativa con diferencias estadísticamente significativas; 10 (216) / 18(6-20), $p<0.01$.

También se encontró un cambio de aptitud en la mayoría de los padres después de la intervención educativa; 77 (83\%) (cuadro III).

En el cuadro IV se puede ver que en la mayoría de los padres se encontró un cambio de aptitud después de la intervención educativa. Algunos padres alcanzaron un valor alto en la escala $n=63(69 \%)$.

Cuadro II

COMPARACIÓN DE LAS CALIFICACIONES ANTES Y DESPUÉS DE LA INTERVENCIÓN EDUCATIVA PARA PREVENIR ABUSO SEXUAL INFANTIL. JALISCO, MÉXICO, DICIEMBRE DE 2009

Aptitud en los padres
valorada por las calificaciones
Indicadores
$n=92$

Antes de la interven;ión educativa con EPP,* mediana (rango) $10(2-12)$

Después de la intervención educativa con EPP, mediana (rango)

Valor de $p$ $18(6-20)$ $<0.01$

Nota: Para comparar la diferencia de medianas antes y después se utilizó la prueba de Wilcoxon

* EPP= Estrategia Promotora Participativa

\section{Cuadro III}

Cambios en la APTITUd de los PAdRes de faMilia DESPUÉS DE LA INTERVENCIÓN EDUCATIVA PARA PREVENIR ABUSO SEXUAL INFANTIL. JALISCO, MÉXICO, DICIEMBRE DE 2009

Cambios en la aptitud de
los padres después de la
intervención educativa
$n=92(\%)$

\begin{tabular}{lc} 
Subieron en relación con la basal & $77(84)$ \\
\hline Bajaron en relación con la basal & $5(5)$ \\
\hline Sin cambios en relación con la basal & $10(\mathrm{II})$ \\
\hline Valor de $p$ & $<0.0 \mathrm{I}$
\end{tabular}

Nota: Para la comparación entre grupos de las variables cualitativas se utilizó la prueba de ji ${ }^{2}$ 


\section{Cuadro IV}

ESCALA DE LA APTITUD EN PADRES DE FAMILIA ANTES Y DESPUÉS DE LA ESTRATEGIA EDUCATIVA PARTICIPATIVA PARA PREVENIR ABUSO SEXUAL INFANTIL. JalisCo, MÉXICO, DICIEMBRE DE 2009

\begin{tabular}{lcc} 
& & \multicolumn{2}{c}{$\begin{array}{c}\text { Aptitud en los padres } \\
\text { Escala }\end{array}$} & \multicolumn{2}{c}{ Intervención participativa } \\
\cline { 2 - 4 } & $\begin{array}{c}\text { Antes } \\
n=92(\%)\end{array}$ & $\begin{array}{c}\text { Después } \\
n=92(\%)\end{array}$ \\
Muy alto $\quad 17-20$ & $0(0)$ & $5(5)$ \\
\hline Alto $\quad 14-16$ & $2(2)$ & $63(69)$ \\
\hline Medio $\quad 11-13$ & $7(8)$ & $9(10)$ \\
\hline Bajo $\quad 8-10$ & $38(41)$ & $10(1 \mathrm{I})$ \\
\hline Muy bajo $4-7$ & $45(49)$ & $5(5)$ \\
\hline Azar $\quad 0-3$ & $0(0)$ & $0(0)$
\end{tabular}

Nota: Se utilizó frecuencias y porcentajes para variables cualitativas

En el cuadro V se muestra el porcentaje de padres que identificaron los conceptos sobre abuso sexual infantil antes / después (incremento en porcentaje). Después de la intervención educativa se encontró una mayor proporción de padres que identificaron que el abuso sexual infantil es frecuente $40 / 88 \%$ (48\%); se encuentra presente la personalidad del agresor $20 / 78 \%$ (58\%) y de víctima 10/75\% (65\%). También se identificaron otras características presentes en este tipo de abuso.

\section{Discusión}

En el presente estudio, después de la intervención educativa con la estrategia promotora de la participación, se encontró un mayor aprendizaje con cambio de aptitud en los padres sobre el impacto de una educación con equidad en los menores para prevenir el ASI.

Estos datos son importantes en el contexto de la educación. Demuestran la importancia de la participación activa de los padres en el desarrollo de habilidades para comprender temas específicos, deducir información y llegar a conclusiones relevantes por medio de la comprensión de los mismos. Esto ayudará a resolver problemas y tomar mejores decisiones.

En esta investigación, la construcción del instrumento de medición utilizado para evaluar el cambio de aptitud en los padres fue revisada y aprobada por expertos en educación y sexología educativa infantil. Posteriormente se aplicó en un grupo piloto de padres y se encontró un coeficiente de confiabilidad alto. Por lo tanto, el instrumento tenía los criterios de validez y confiabilidad.

En la literatura médica no se encontró ninguna evaluación de intervención educativa con la EPP en los padres sobre el tema de educar con equidad a los

CuadroV

APtitud en los PADRES ANTES y DESPUÉS de LA INTERVENCIÓN EDUCATIVA PARA PREVENIR ABUSO SEXUAL INFANTIL. JALISCO, MÉXICO, DICIEMBRE DE 2009

\begin{tabular}{|c|c|c|}
\hline \multirow[b]{2}{*}{ Indicadores } & \multicolumn{2}{|c|}{ Frecuencia en las respuestas de los padres } \\
\hline & Antes $n=92$ & Después $n=92$ \\
\hline $\begin{array}{l}\text { Abuso sexual infantil } \\
\text { Es frecuente, } n(\%)\end{array}$ & $40(44)$ & $88(96)$ \\
\hline Se encuentra la personalidad agresiva, $n(\%)$ & $20(22)$ & $78(85)$ \\
\hline Se encuentra la personalidad de víctima, (\%) & $10(\mathrm{II})$ & $75(82)$ \\
\hline La personalidad se forma & & \\
\hline En los primeros años de vida, $n(\%)$ & $12(13)$ & $84(92)$ \\
\hline Los padres influyen en su formación, $n$ (\%) & $53(57)$ & $88(96)$ \\
\hline En la educación con equidad todos los niños & & \\
\hline Tienen derecho a estudiar, $(\%)$ & $82(89)$ & $92(100)$ \\
\hline Derecho a realizar las mismas actividades, $n(\%)$ & $12(13)$ & $90(98)$ \\
\hline Tener las mismas obligaciones, $n(\%)$ & $14(15)$ & $89(97)$ \\
\hline Recibir las mismas sanciones, $n(\%)$ & $25(27)$ & $87(95)$ \\
\hline
\end{tabular}

Nota: Se utilizaron frecuencias y porcentajes para variables cualitativas 
menores para prevenir el abuso sexual infantil. La mayoría de las intervenciones educativas sobre ASI son dirigidas hacia los menores. ${ }^{19-21}$ Sin embargo, existen limitaciones con estas intervenciones, como la pasividad del alumno y el hecho de que el aprendizaje sólo queda en la memoria.

Algunos autores describen que en la estrategia promotora de la participación los alumnos tienen una participación activa; el aprendizaje se inicia con la búsqueda de la información, posteriormente se transforma al compartir experiencias, se crea una discusión y finalmente se realizan conclusiones. El profesor es un facilitador que favorece un ambiente de reflexión con el propósito de que el alumno integre la teoría con las experiencias, lo que incrementa los resultados del aprendizaje. $^{18,26}$

Trujillo y colaboradores, ${ }^{27}$ en un estudio longitudinal realizado en 88 internos de pregrado, evaluaron la aptitud clínica antes y después de recibir una intervención educativa. Ellos encontraron un mayor aprendizaje, demostrado al encontrar mejores puntajes después de la intervención educativa con diferencias estadísticamente significativas. Los autores mencionan que la teoría no es suficiente para el aprendizaje, sino que es necesario el desarrollo de aptitudes para ser mejores médicos, lo cual se puede lograr con una adecuada estrategia educativa participativa.

Otros autores evaluaron una intervención educativa con la estrategia promotora participativa sobre el aprendizaje antes y después para controlar la presión arterial media y el índice de masa corporal en los pacientes. ${ }^{28}$ Se encontró un mayor aprendizaje con cambio de aptitud en los pacientes al disminuir las cifras de la presión arterial y el índice de masa corporal.

Resultados similares fueron encontrados en esta investigación al encontrar después de la intervención educativa con la estrategia promotora participativa un mayor aprendizaje en los padres, lo cual se demostró con un incremento en el puntaje de las respuestas en relación con el valor basal con diferencias estadísticamente significativas sobre el tema impartido: Impacto de educar con equidad a los menores para prevenir el ASI.

Dentro de las limitaciones de este estudio está el hecho de que los padres de esta investigación tenían diferentes edades y grados de escolaridad, característica que influye en el aprendizaje. ${ }^{29}$ Otra limitación fue no contar con un grupo de comparación, lo cual hubiera permitido estimar con mayor fortaleza los resultados encontrados en esta investigación.

Una de las ventajas para utilizar la estrategia promotora participativa es que no se requiere de un material costoso ni especializado. Dentro de las desventajas se encuentra el hecho de que el profesor sea capacitado para aplicar este tipo de estrategia educativa. Esta investigación es importante por la aplicación que puede tener en la educación para lograr un mayor aprendizaje.

\section{Conclusiones}

Después de la intervención educativa con la estrategia promotora participativa dirigida a los padres con menores en educación preescolar, se encontró un mayor aprendizaje, lo cual cambió la aptitud sobre el impacto de educar con equidad a los menores para prevenir abuso sexual infantil. Es necesario realizar futuras investigaciones para corroborar los resultados encontrados en esta investigación. Además, es importante compararla con otras estrategias educativas.

\section{Agradecimientos}

Los autores agradecen a la Secretaría de Educación Pública de la localidad en donde se llevó a cabo el estudio y al Instituto Mexicano de Estudios Pedagógicos por la ayuda desinteresada para la realización de esta investigación, así como a los padres de familia por su participación.

Declaración de conflicto de intereses: Los autores declararon no tener conflicto de intereses.

\section{Referencias}

I. Who Global Estimates of Health Consequences Due to Violence against Children. Background Paper to the Secretary-General's Study on Violence Against Children. Geneva:WHO, 2006.

2. Rivera-Rivera L,Allen B,Chávez-Ayala R, Ávila-Burgos L.Abuso físico y sexual durante la niñez y revictimización de las mujeres mexicanas durante la edad adulta. Salud Publica Mex 2006;48:268-278.

3. Cloitre M, Cohen LR, Edelman RE, Han H. Posttraumatic stress disorder and extent of trauma exposure as correlates of medical problems and perceived health among women with childhood abuse. Women Health 2001;34(3): I- 17.

4. Pérez-Amezcua B, Rivera-Rivera L,Atienzo EE, De Castro F, LeyvaLópez A, Chávez-Ayala R. Prevalencia y factores asociados a la ideación e intento suicida en adolescentes de educación media superior de la República mexicana. Salud Publica Mex 2010;52(4):324-333. 5. Drossman DA, Talley NJ, Leserman J, Olden KW, Barreiro MA. Sexual and physical abuse and gastrointestinal illness: review and recommendations. Ann Intern Med 1995; 123:782-794.

6. Flannery DJ, Singer MI,Wester K.Violence exposure, psychological trauma, and suicide risk in a community sample of dangerously violent adolescents. J Am Acad Child Adolesc Psychiatry 200 I;40(4):435-442. 7. Hill J, Pickles A, Burnside E, Byatt M, Rollinson L, Davis R et al. Child sexual abuse, poor parental care and adult depression: evidence for different mechanisms. Br J Psychiatry 2001;179:104-109.

8.American Academy of Pediatrics. Guidelines for the Evaluation of Sexual Abuse of Children. Pediatrics 1999;3:186-190. 
9. Perea-Martínez A, Loredo-Abdalá A, Monroy-Villafuerte A, Güicho Alba. El abuso sexual: del silencio Ignominioso a una realidad estigmatizante: Maltrato en Niños y adolescentes. México: Editores de Textos Mexicanos, 2004:75-102

10. Cantón Duarte J, Cortés Arboleda MR. Guía para la evaluación del abuso sexual infantil. Madrid: Editorial Pirámide, 2000.

II. Reichborn-Kjennerud T. Genetics of personality disorders. Clin Lab Med 2010;30(4):893-910.

12. Hopwood C], Morey LC, Markowitz JC, Pinto A, Skodol AE, Gunderson $\mathrm{JG}$, et al.The construct validity of passive-aggressive personality disorder. Psychiatry 2009;72(3):256-267.

13. Perry BD, Mann D, Corell A P, Ludy-Dobson C. Child Physical Abuse 2002;I:197-20I.

14. Cieslak R, Benight CC, Caden Lehman V. Coping self-efficacy mediates the effects of negative cognitions on posttraumatic distress. Behav Res Ther 2008;46:788-798.

15. Chávez Ayala R, Rivera-Rivera L,Angeles-Llerenas A, Díaz-Cerón E,Allen-Leigh B, Ponce EL. Factors for sexual abuse during childhood and adolescence in students of Morelos, Mexico. Salud Publica Mex 2009;43:506-5I4.

16. Vung ND, Krantz G.Childhood experiences of interparental violence as a risk factor for intimate partner violence: a population-based study from northern Vietnam J Epidemiol Community Health 2009;63(9):708-7I4.

17. Tyer-Viola LA, Cesario SK.Addressing poverty, education, and gender equality to improve the health of women worldwide.J Obstet Gynecol Neonatal Nurs 201;39(5):580-589.

18. Daro D. Prevention of childhood sexual abuse. Future child 1994:4:198-223.
19. Cox A. Preventing child abuse:A review of community- based projects. I: Intervening on processes and outcomes of reviews. Child Abuse 1997:6:243-256.

20. Moody EE. Current trends and sigues in childhood sexual abuse prevention programs. Elementary School Guidance and Counseling 1994;28: 25I-256.

21. Finkelhor D. The prevention of childhood sexual abuse. Future Child 2009;19:169-194

22. Viniegra VL. La investigación en educación: papel de la teoría y de la observación. Rev Med IMSS 1999;35-50.

23. Viniegra L. El camino a la crítica. En materiales para la crítica de la educación. Rev Med IMSS 1998: I-50.

24. Viniegra L, Jiménez J, Pérez R. El desafío de la evaluación de la competencia clínica. Rev Invest Clin 1991;45:87-95.

25. World Medical Association. World Medical Association Declaration of Helsinki. Ethical principles for medical research involving human subjects. Nurs Ethics 2002;9(I):105-109

26. Viniegra L, Jiménez J. Nuevas aproximaciones a la medición de la competencia clínica. Rev Invest Clin 1992;44:269-275.

27. Trujillo F, González R, Murguía C. Competencia clínica en diabetes mellitus. Rev Med IMSS 2002; 40(6):467-472.

28. Peredo-Rosado P, Gómez-López VM. Impacto de una estrategia educativa Activa participativa en el control de la hipertensión arterial. Rev Med IMSS 2005;43(2) I25-I29.

29. Benedit MJ, Martínez-Arias R. Diferencias con la edad en el uso de estrategias, en el aprendizaje y en la retención. Anales de psicología 1998;14:39-156. 